\title{
BMJ Open PRedicting Outcomes For Crohn's dIsease using a moLecular biomarkEr (PROFILE): protocol for a multicentre, randomised, biomarker-stratified trial
}

\author{
Miles Parkes, ${ }^{1}$ Nurulamin M Noor, ${ }^{1}$ Francis Dowling, ${ }^{2}$ Harvey Leung, ${ }^{2}$ \\ Simon Bond, ${ }^{2,3}$ Lynne Whitehead, ${ }^{4}$ Sara Upponi, ${ }^{5}$ Paul Kinnon, ${ }^{6}$ \\ Andrew P Sandham, ${ }^{6}$ Paul A Lyons, ${ }^{1,6}$ Eoin F McKinney, ${ }^{1,6}$ Kenneth G C Smith, ${ }^{1,6}$ \\ James C Lee ${ }^{1}$
}

To cite: Parkes M, Noor NM Dowling F, et al. PRedicting Outcomes For Crohn's dlsease using a moLecular biomarkEr (PROFILE): protocol for a multicentre, randomised, biomarkerstratified trial. BMJ Open 2018;8:e026767. doi:10.1136/ bmjopen-2018-026767

- Prepublication history and additional material for this paper are available online. To view these files, please visit the journal online (http://dx.doi org/10.1136/bmjopen-2018026767).

MP and NMN contributed equally.

Received 19 September 2018 Revised 8 October 2018 Accepted 10 October 2018

Check for updates

(C) Author(s) (or their employer(s)) 2018. Re-use permitted under CC BY. Published by BMJ.

For numbered affiliations see end of article.

Correspondence to Dr Miles Parkes; miles.parkes@addenbrookes. nhs.uk and Dr James C Lee; jcl65@cam.ac.uk

\section{ABSTRACT}

Background The course of Crohn's disease (CD) varies substantially between individuals, but reliable prognostic markers do not exist. This hinders disease management because patients with aggressive disease are undertreated by conventional 'step-up' therapy (in which treatment is gradually escalated in response to refractory or relapsing disease) while those with more indolent disease would be exposed to unnecessary treatment-related toxicity if a more aggressive 'top-down' approach was indiscriminately used. The Predicting outcomes for Crohn's disease using a molecular biomarker trial will assess whether a prognostic transcriptional biomarker, that we have developed and validated, can improve clinical outcomes by facilitating personalised therapy in $\mathrm{CD}$. This represents the first the biomarker-stratified trial in inflammatory bowel disease. Methods and analysis This biomarker-stratified trial will compare the relative efficacy of 'top-down' and 'accelerated step-up' therapy between biomarkerdefined subgroups of patients with newly diagnosed $C D$. 400 participants from $\sim 50$ UK centres will be recruited. Subjects within each biomarker subgroup (IBD ${ }^{\text {hi }}$ or IBD ${ }^{10}$ ) will be randomised (1:1) to receive one of the treatment strategies until trial completion (48 weeks). The primary outcome is the incidence of sustained surgery and steroid-free remission from the completion of induction treatment through to week 48 . Secondary outcomes include mucosal healing, quality-of-life assessments and surrogate measures of disease burden including number of flares, cumulative steroid exposure, number of hospital admissions and number of Crohn's-related surgeries (assessed hierarchically). Analyses will compare the relative benefit of the treatment strategies in each biomarker-defined subgroup, powered as an interaction analysis, to determine whether the biomarker can accurately match patients to the most appropriate therapy. Ethics and dissemination Ethical approval has been obtained and recruitment is under way at sites around the UK. Following trial completion and data analysis, the results of the trial will be submitted for publication in peer-reviewed journals and presented at international conferences.

Trial registration number ISRCTN11808228; Pre-results.

\section{Strengths and limitations of this study}

- The first biomarker-stratified trial in inflammatory bowel disease, comparing the relative benefit of 'top-down' over 'accelerated step-up' therapy in biomarker-defined subgroups of patients with newly diagnosed Crohn's disease.

- The largest interventional trial ever conducted in adult patients with newly diagnosed Crohn's disease, incorporating 400 patients across approximately 50 sites.

- Findings have the potential to demonstrate that personalised therapy can be effectively delivered to patients with Crohn's disease at the time of diagnosis using a blood-based prognostic biomarker.

- Study limited to the UK

- Top-down therapy limited to treatment with infliximab and an immunomodulator (which may be superseded by other treatments in the future).

\section{INTRODUCTION}

Crohn's disease (CD) is a relapsing-remitting form of inflammatory bowel disease (IBD) that can affect any part of the intestine, most commonly the ileum and/or colon. It is a common condition, affecting $\sim 1$ in 400-500 people in Northwestern Europe and North America, with a steadily rising global incidence. $^{12}$

Like many other immune-mediated diseases, the course of $\mathrm{CD}$ varies substantially between affected individuals, but no reliable prognostic markers currently exist. The most common treatment strategy in CD is therefore based on a reactive, stepwise escalation in therapy that occurs in response to recurrent flares or persistently active disease. This approach (termed 'step-up') should not overtreat patients but will inevitably expose some individuals to cumulative intestinal damage 
and disease-related complications while therapies that are insufficiently potent for them are trialled.

In 2008, it was shown that early use of anti-tumour necrosis factor $\alpha(\mathrm{TNF} \alpha)$ monoclonal antibodies (anti$\mathrm{TNF} \alpha$ therapy) was superior to conventional step-up management. ${ }^{3}$ Further support for early anti-TNF $\alpha$ use came from registration trials, which demonstrated greater efficacy of anti-TNF $\alpha$ therapy when it was used earlier in the disease course ${ }^{45}$; and the SONIC trial, which showed that combining anti-TNF $\alpha$ (infliximab) with azathioprine (termed combination or 'top-down' therapy) achieved results superior to either alone. ${ }^{6}$ However, it is widely recognised that the indiscriminate use of combination therapy in all patients would expose those patients destined for mild disease to the risks and side-effects of treatment that their disease did not require, and would also be economically unfeasible.

In an attempt to reconcile these issues, subsequent trials have sought to identify approaches that could still deliver relatively early, aggressive therapy but also be economically feasible. The Randomised Evaluation of an Algorithm for Crohn's Treatment (REACT) trial, for example, investigated whether accelerating more quickly up the treatment ladder ('accelerated step-up') would lead to better outcomes. ${ }^{7}$ Similarly, the AZathioprine for Treatment or Early Crohn's disease in adults (AZTEC) and Résultat de l'Adjonction Précoce d'ImmunoDépresseurs (RAPID) trials investigated whether initiating azathioprine, a less potent but cheaper immunomodulator, in all patients at diagnosis would improve outcomes. ${ }^{89}$ However, none of these studies have demonstrated improved efficacy over standard care, leading many to conclude that a 'precision' (or 'personalised') approach would be required in which the most potent treatments are targeted to those who need them. Unfortunately, despite investigation into the prognostic utility of clinical, genetic and serological markers, there remain no well-validated prognostic tools for CD that can reliably predict the disease course from diagnosis. Indeed, a recent priority setting partnership group, tasked with identifying major areas of unmet need in IBD research, designated the need to develop markers to guide treatment for individual patients as the most important unmet need in IBD. ${ }^{10}$ Consistent with this, a survey of 52 US and 50 UK gastroenterologists (commissioned through Apex Healthcare Consulting) showed that nearly all gastroenterologists recognised a need for an assay that could predict the clinical outcome and probability of relapse in CD (UK 98\%, US 94\%; table 1). Moreover, if the results of such a biomarker enabled gastroenterologists to amend their treatment approach, all of the respondents would use the test in their practice (table 1).

Our group has previously identified a gene expression signature in peripheral blood $\mathrm{CD} 8+\mathrm{T}$ cells from patients with active, untreated IBD (and other autoimmune diseases) that is related to $\mathrm{T}$ cell exhaustion and which correlates with subsequent prognosis. ${ }^{11-13}$ Patients in the IBD1 subgroup, defined by this signature, had a much more aggressive disease than those in the IBD2 subgroup, with earlier recurrence of disease and more flares over time. ${ }^{11}$ To help translate this to routine clinical practice, we have since developed a whole blood qPCR assay that can identify patient subgroups which are analogous to those identified by the CD8 signature, but which does not require cell separation (manuscript in preparation). This assay has been independently validated in prospectively-collected cohorts of UC and CD patients from four centres around the UK. ${ }^{14}$ We now propose to conduct a biomarker-stratified trial to determine whether this biomarker can facilitate the delivery of personalised medicine in $\mathrm{CD}$ and improve outcomes.

This manuscript summarises the approved PRedicting Outcomes For Crohn's dIsease using a moLecular biomarkEr (PROFILE) trial protocol that is in use at the time of publication (V.3.0, 30 April 2018). The full version of the protocol is available at: http://www. crohnsprofiletrial.com/index.php/investigators/ downloads/.

The PROFILE trial participant information sheet (PIS) that is in use at the time of publication (V.3.1, 25 June

Table 1 Summary results of an independent 2015 survey of practising gastroenterologists performed by Apex Healthcare Consulting

\begin{tabular}{|c|c|c|}
\hline & UK $(n=50)$ & USA ( $n=52)$ \\
\hline 'CD patients are at moderate-to-high risk of relapse throughout their lives' & Agree-80\% (40) & Agree-79\% (41) \\
\hline $\begin{array}{l}\text { 'There is a need for an assay that would predict clinical outcome and probability of } \\
\text { relapse in CD' }\end{array}$ & Agree-98\% (49) & Agree-94\% (49) \\
\hline $\begin{array}{l}\text { Would you use a test to predict clinical outcome and probability of relapse even if you } \\
\text { could not change your treatment approach? }\end{array}$ & Yes-58\% (29) & Yes-54\% (28) \\
\hline $\begin{array}{l}\text { How many days following a test to predict clinical outcome and probability of relapse } \\
\text { would you require the results for this to be useful? }\end{array}$ & 10 days (mean) & 9 days (mean) \\
\hline
\end{tabular}

Gastroenterologists: clinically active attending physicians (USA) or consultants (UK) with 5-30 years specialty experience, including IBD caseload. Survey funded by Wellcome Trust (Interim Translational Award 099450/Z/12/Z).

$\mathrm{CD}$, Crohn's disease; IBD, inflammatory bowel disease. 


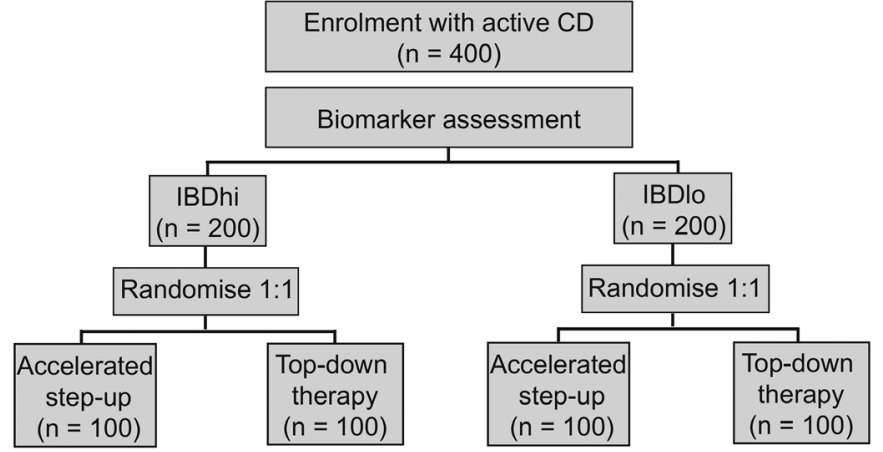

Figure 1 Trial design. Following biomarker stratification, patients will be randomised in a 1:1 fashion to either 'topdown' or 'accelerated step-up' treatment arms. CD, Crohn's disease; IBD, inflammatory bowel disease.

2018) is available at: http://www.crohnsprofiletrial.com/ index.php/participants/downloads/.

Any future amendments to this protocol or PIS will require agreement with the sponsors and amendments will only be initiated following approval by a Research Ethics Committee.

\section{Aims and objectives}

The PROFILE trial will test whether stratification using a whole blood gene expression biomarker can facilitate personalised therapy in $\mathrm{CD}$ and improve clinical outcomes. The hypothesis is that the biomarker will identify individuals destined to run an aggressive, relapsing course, and that in these individuals a greater benefit of early top-down therapy will be observed. Similarly, we hypothesise that the biomarker will reliably identify those patients destined to experience more indolent disease, who can be effectively managed using conventional accelerated step-up approaches without the risks and side-effects of unnecessary immunosuppression.

In addition, the trial will seek to advance scientific understanding of $\mathrm{CD}$ through the collection of a range of biological samples for future exploratory translational and scientific studies. These will include microbial, metabolomic, proteomic, genetic and transcriptomic samples.

\section{Methods and analysis}

\section{Trial design and flow chart}

The trial is designed as a randomised, biomarker-stratified trial to assess the relative benefit of different treatment approaches in biomarker-defined subgroups. This is an established design for the validation of predictive biomarkers, ${ }^{15}$ and has been used widely in the setting of oncology trials. ${ }^{16}$ Within each biomarker group, patients will be randomised in a 1:1 ratio to receive either top-down or accelerated step-up therapy (figure 1).

\section{Trial sites}

PROFILE is a multicentre trial based in National Health Service hospitals within the UK. This trial aims to recruit 400 participants with newly diagnosed $\mathrm{CD}$ and will be

\section{Box 1 Eligibility criteria for the PROFILE trial}

\section{Inclusion criteria}

Subjects meeting all of the criteria below may be included in the trial:

- CD diagnosed within 3 months using standard endoscopic, histological or radiological criteria*.

- Clinical evidence of active Crohn's disease (CD) (corresponding to Harvey Bradshaw Index $>7$ ).

- Endoscopic evidence of at least moderately active CD (corresponding to Simplified Endoscopic Score in $\mathrm{CD}>6$ or $>4$ if limited to the terminal ileum).

- C reactive protein>upper limit of normal on local assay or faecal calprotectin $>200 \mu \mathrm{g} / \mathrm{g}$.

- Immunomodulator and anti-TNF $\alpha$ treatment naïve†.

- Aged 16-80 years old.

\section{Exclusion criteria}

The presence of any of the following would preclude patient inclusion:

- Patients with ulcerative colitis.

- Patients with fistulating perianal CD or active perianal sepsis.

- Patients with obstructive symptoms and evidence of a fixed stricture on radiology or colonoscopy, which suggest that the subject is at high risk of requiring surgery over the following year.

- Patients with contraindications to trial medications.

- Patients who are pregnant or breast feeding at baseline.

- Other serious medical or psychiatric illness currently ongoing, or experienced in the last 3 months, that could compromise the trial.

- Patients unable to comply with protocol requirements (for reasons including alcohol and/or recreational drug abuse).

*Newly diagnosed patchy colonic inflammation, initially diagnosed as indeterminate colitis, would meet inclusion criteria if clinical impression consistent with $\mathrm{CD}$.

†Patients need to have discontinued systemic corticosteroids for 1 week or more prior to screening assessments and still have ongoing, active disease.

conducted in approximately 50 sites (http://www.crohnsprofiletrial.com/index.php/investigators/).

\section{Trial duration}

After providing informed consent, participants will be enrolled within the trial for 48 weeks following the baseline visit. There will be a total of six mandatory trial visits, during which data will be collected. These will take place at the same timepoints for all participants and have been timed to coincide with infliximab infusion visits where possible (for those receiving top-down therapy). The end of the trial will be the last participant's last visit.

\section{Eligibility criteria}

Patients will be considered eligible for enrolment if they fulfil all of the inclusion criteria and meet none of the exclusion criteria (box 1). The target population are patients with newly diagnosed, active $\mathrm{CD}$ who are immunomodulator and anti-TNF $\alpha$ treatment naïve.

\section{Patient and public involvement}

The development and advancement of personalised medicine in CD represents a major goal for both patients and physicians, and was recently named one of the key research priorities in IBD by a priority setting partnership 
group, which included both patients and other key stakeholders. ${ }^{10}$

A local panel of patients with $\mathrm{CD}$ at Cambridge University Hospitals National Health Service Trust was actively involved in the design of the study and development of study documentation, and feedback was also obtained by a broader panel of non-IBD patients convened by the Cambridge Clinical Trials Unit. Patient support groups (Crohn's and Colitis UK) were engaged during the conduct of the trial via invitation to investigator meetings, presentation to patient support groups, and publicity of the trial on their website and social media platforms. Both Crohn and Colitis UK and trial participants have also contributed to the content of the trial website (http://www.crohnsprofiletrial.com/index.php/participants), although patients were not directly involved in the recruitment to, or conduct of, the trial.

Following trial completion and reporting, results of the trial will be disseminated in an easy-to-understand format to all trial participants and to Crohn's and Colitis UK, as well to the general public via press releases and the public engagement team at the University of Cambridge.

\section{Outcome measures \\ Primary outcome}

Incidence of sustained surgery and steroid-free remission from the completion of induction treatment (a standard, 8-week course of oral steroids) through to week 48. (Remission=Harvey Bradshaw Index $(\mathrm{HBI})<4$. Requirement for a course of systemic glucocorticoids for active $\mathrm{CD}$ would result in failure to meet the primary outcome measure.)

\section{Secondary outcomes}

1. Mucosal healing (assessed using Simplified Endoscopic Score in CD (SES-CD)).

2. Quality-of-life assessment (assessed using IBD Questionnaire).

3. Assessment of cumulative disease burden based on:

i. Number of flares by 1 year.

ii. Cumulative glucocorticoid exposure by 1 year.

iii. Steroid-free remission by 1 year.

iv. Number of hospital admissions and CD surgeries by 1 year.

\section{Health economic evaluation}

During the course of the trial, there will be a local health economic analysis conducted by the Cambridge Centre for Health Services Research, as well as a national health economic analysis conducted by the National Institute for Health and Clinical Excellence. The findings of these health economic analyses will be disseminated alongside clinical trial findings.

\section{Treatment assignment}

All patients considered eligible for the trial at the screening visit will have an 8-week reducing course of prednisolone initiated for treatment of their active luminal CD following screening assessments. Each will be assigned a unique participant ID number, for which a biomarker result will be returned. Anonymised data on all participants who are approached will be collated in accordance with Consolidated Standards of Reporting Trials guidelines. Following biomarker assessment, participants in each biomarker subgroup will be randomly assigned (1:1) to either top-down or accelerated step-up therapy, using a computer-generated algorithm (figure 1). This will occur within 14 days of screening (plus or minus 5 days).

As the trial is testing the ability of the biomarker to stratify therapy, rather than the efficacy of the individual medications (which are established treatments for $\mathrm{CD}$ ), PROFILE has been designated a non-clinical trial of investigational medicine product. All treatments will be open label, but clinicians and participants will be blinded to biomarker subgroup designation.

\section{Treatment arms}

Following induction treatment with prednisolone, patients will follow the treatment strategy to which they are randomised. These are:

\section{Accelerated step-up therapy}

- Flare 1 (after induction therapy or if disease reflares during induction therapy): commence azathioprine $(2.5 \mathrm{mg} / \mathrm{kg})$ or low-dose 6-mercaptopurine with allopurinol (if mild intolerance to azathioprine) or methotrexate (if severe intolerance to thiopurines or thiopurine methyltransferase (TPMT) null) together with a 12-week reducing course of prednisolone.

- Flare 2: commence infliximab. If suboptimal response, then for infliximab dose escalation as outlined in the full trial protocol.

- Flare 3+ (ie, disease flare after infliximab dose optimisation): 8-week reducing course of prednisolone.

\section{Top-down therapy}

- Infliximab started 2 weeks after randomisation with azathioprine $(2.5 \mathrm{mg} / \mathrm{kg})$ or alternative immunomodulator as described above. If suboptimal response, then for infliximab dose escalation as described in the full trial protocol. The rate of weaning of prednisolone should be accelerated once infliximab is given to $10 \mathrm{mg} /$ week.

- Subsequent disease flares (ie, disease flare after infliximab dose optimisation): 8-week reducing course of prednisolone.

Participants with persistent non-response to infliximab can have early treatment termination and revert to standard care, at the discretion of their local clinical team.

\section{Trial procedures and assessments}

Newly diagnosed patients with $\mathrm{CD}$ will be recruited from a predominantly outpatient setting. Potential trial patients will be identified by local clinical team members and be given a PIS prior to attending a screening visit. All participants must have had a colonoscopy before screening, 


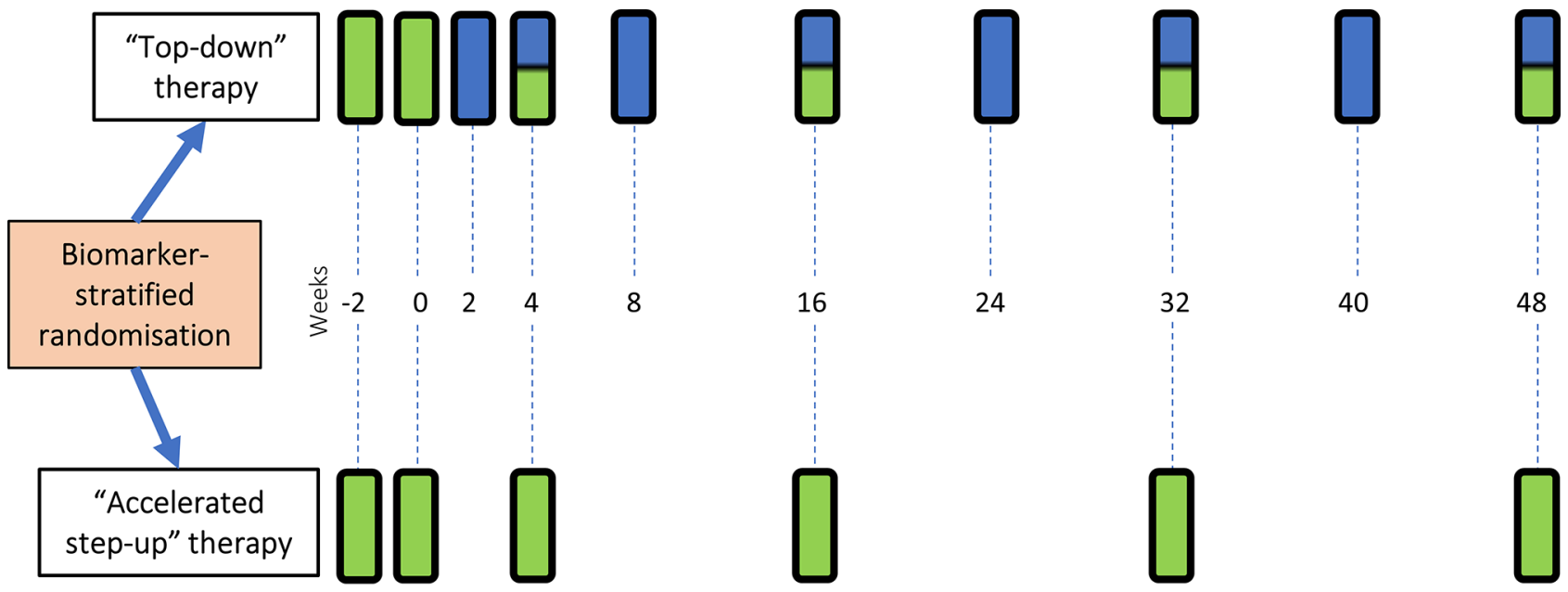

Scheduled trial visit

Scheduled infusion visit

Figure 2 Trial visits for participants. Patients randomised to 'accelerated step-up' will have a total of five further trial visits after their initial screening visit. Participants randomised to the 'top-down' group will be started on infliximab at week 2. All further infliximab infusion visits should be aligned to scheduled trial visits wherever possible in order to minimise visit burden for participants. Participants in the top-down group will also have five trial visits and will also attend hospital an additional four times for infliximab infusions. Randomisation occurs at week 0.

where possible recorded for central reading. A magnetic resonance enterography (MRE) to stage disease in accordance with European consensus guidelines ${ }^{17}$ is also required but can be performed after trial entry.

Assessments, data collection and obtaining informed consent will be performed by appropriately trained research staff, as delegated by the principal investigator at each site. At trial visits, clinical data will be collected as well as samples for local and central processing-collection, evaluation and storage of these samples are outlined in the full trial protocol. Participants receiving infliximab should have infusion visits aligned with trial visits, as shown in figure 2, to reduce visit burden and the placebo effect associated with extra visits. ${ }^{18}$ Following their final trial visit, participants will return to normal standard of care, according to local clinical practice.

Only adverse events (AEs) that relate to $\mathrm{CD}$, drug therapy for CD (sufficiently severe to require a change of treatment), or the biomarker sample collection will be recorded and assessed. Safety reporting and assessment of causality and expectedness of serious AEs will occur within standard timelines. The trial sponsors will arrange insurance for negligent harm caused as a result of protocol design and for non-negligent harm arising through participation in the clinical trial.

\section{Sample size calculation}

We will recruit 400 participants into the PROFILE trial. This sample size was determined using a power calculation in which power was calculated by simulating 10000 study designs and counting how many times a significant result was obtained. This was based on previously published remission rates for the primary endpoint, ${ }^{3} 7$ the observed ratio of the $\mathrm{IBD}^{\text {hi }} / \mathrm{IBD}^{\text {lo }}$ biomarker result in existing cohorts $(1: 1)$ and the observed remission rates in each of these cohorts. ${ }^{14}$

\section{Statistical procedures and data analysis plan}

The primary analysis is powered as an interaction analysis, where the interaction refers to the difference between the relative benefit of top-down over accelerated step-up in each subgroup. This analytical strategy maximises the information available from each subgroup, and will determine whether the biomarker can accurately match patients to the most appropriate treatment strategy. Assuming an interaction of 0.3 , a sample size of 346 will provide $90 \%$ power (estimated with $95 \%$ CIs and tested at a two-tailed, $5 \%$ significance level). To allow for a $13.5 \%$ drop out rate, 400 participants will be recruited across approximately 50 sites. This will require recruitment of $\sim 4$ participants per site per year, which is a rate consistent with previous recruitment to investigator-led IBD studies in the UK. ${ }^{19}$ Recruitment began in December 2017.

To control for multiple testing, we will perform a closed testing procedure over the primary and six secondary endpoints, testing the biomarker-treatment interaction. A well-described methodology combining gate-keeping and Holm-Bonferroni methods in formal hypothesis testing will be used, ${ }^{20}$ as outlined in online supplementary figure 1 . The secondary outcome measures will include an endoscopic assessment of mucosal healing (in addition to further analyses using MRE data), a quality-of-life assessment and a third outcome measure related to overall burden of disease (this hierarchically includes number of flares, cumulative steroid exposure, number of hospital admissions and number of Crohn's-related surgeries). 
Mucosal healing has been associated with improved long-term outcomes in $\mathrm{CD} .{ }^{2122}$ The use of central reading, in which the endoscopic images or video recordings are externally evaluated, has been further associated with a reduction in placebo response rates, ${ }^{23}$ in part due to more stringent application of inclusion criteria and assessment of endoscopic response. ${ }^{24}$ The PROFILE trial will use video recording of colonoscopy at the end of the trial period in all patients and at the outset in as many patients as possible, using the SES-CD, ${ }^{25}$ a scoring tool that has been shown to have high inter-rater and intrarater reliability. ${ }^{26}$ To date, many trials using endoscopic endpoints have applied post hoc analyses in small cohorts, resulting in limited power to detect effects. ${ }^{27}$ In this respect, the PROFILE trial will be one of the largest trials to analyse mucosal healing routinely and the first to do so in the setting of adults with CD treated with top-down therapy from diagnosis.

An MRE will be performed at the end of the trial period in all patients. There is increasing interest in the use of MRE as a measure of disease activity in clinical trials, with the development of imaging scores such as the Magnetic Resonance Index of Activity (MaRIA).$^{28} 29$ This, and other similar scores, have often been validated and refined in relatively small cohorts ${ }^{30}$ and none are in routine clinical use. With 400 participants, the PROFILE trial will enable further evaluation of the MaRIA score both in terms of confirming treatment response and as an evaluative index. $^{31}$

Quality-of-life assessments will be performed over repeated visits and will be analysed using a mixed effect repeat measure analysis with a clustered patient-level residual error with unstructured covariance over visits, fixed effects for visit and all other covariates assumed to have a constant fixed effect over time.

It is anticipated that future data collection will also take place following completion of treatment to assess disease burden and the longer term impact of top-down versus accelerated step-up treatment approaches on subsequent disease course for these patients.

\section{CONCLUSIONS}

Currently, there is a clear unmet need in the management of IBD, in that treatment strategies-whatever they may be-are typically applied in a one-size-fits-all manner or using 'prognostic' markers that have not been shown to be able to guide therapy.

The PROFILE trial is the first biomarker-stratified trial in IBD and will investigate whether a blood-based biomarker, assessed at diagnosis, can stratify patients with $\mathrm{CD}$ to receive therapy that is appropriately matched to their subsequent disease course.

If stratification by $\mathrm{IBD}^{\text {hi }} / \mathrm{IBD}^{\text {lo }}$ status is demonstrated to improve clinical outcomes by appropriately identifying those patients who require top-down therapy and those who can be safely managed with accelerated step-up therapy, this would represent a step change in the management of $\mathrm{CD}$ and would help make personalised medicine a reality for patients.

\section{Ethics and dissemination}

Recruitment for the PROFILE trial began in December 2017 and is currently ongoing at sites around the United Kingdom. On completion of the trial, the data will be analysed and tabulated and a final trial report prepared. Following trial completion and analysis, the results will be presented at scientific meetings and submitted for publication in a peer-reviewed journal. Press releases will be prepared to accompany publication of this trial in order to share the results more widely with the global medical community, trial participants and patient support groups. Reasonable applications for individual clinical trial participant-level data will be considered by the trial team and shared on a controlled access basis if approved. Authorship of final trial outputs will be assigned in accordance with guidelines set out by the International Committee of Medical Journal Editors. The Standard Protocol Items: Recommendations for Interventional Trials reporting guidelines have been used in preparation of this article. ${ }^{32}$

\section{Patient records}

Data are collected via a paper case report form, provided by the trial coordination team, and after being input electronically, will be stored in a secured database. Participants will only be identifiable by a trial-specific number in the database. Essential documents will be retained until at least 15 years after the publication of the clinical trial report.

\section{TRIAL COMMITTEES}

The unblinded data will be presented to the Data Monitoring Committee, who will meet on a regular basis throughout the trial and who are independent from the sponsor. The Data Monitoring Committee will then prepare a report for the Trial Steering Committee who will provide overall supervision of the trial.

\section{Author affiliations}

${ }^{1}$ Department of Medicine, University of Cambridge School of Clinical Medicine, Addenbrooke's Hospital, Cambridge, UK

${ }^{2}$ Cambridge Clinical Trials Unit, Cambridge University Hospitals NHS Foundation Trust, Cambridge, UK

${ }^{3}$ Medical Research Council Biostatistics Unit, University of Cambridge, Cambridge, UK

${ }^{4}$ Clinical Trials Pharmacy, Addenbrooke's Hospital, Cambridge, UK

${ }^{5}$ Department of Radiology, Addenbrooke's Hospital, Cambridge, UK

${ }^{6}$ Predictlmmune Ltd, Babraham Research Campus, Cambridge, UK

Acknowledgements The authors are grateful for input and assistance from Carrie Bayliss (Cambridge Clinical Trials Unit) and Amanda Wooding (Cambridge Enterprise) in securing funding for this trial, and to Adrian Mander, Robert Gray, Geert D'Haens, Severine Vermeire, Sharon 0'Byrne, Subrata Ghosh, Nick Carroll and Ed Godfrey for helpful discussion regarding the protocol. They are grateful to the patients who provided feedback on the study design and documentation. The protocol has been peer reviewed by the British Society of Gastroenterology IBD Clinical Research Group. Details of participating centres can be found at http://www.crohnsprofiletrial.com/index.php/investigators/. KGCS is a Wellcome Investigator and an NIHR Senior Investigator. 
Contributors MP, NMN, FD, KGCS and JCL wrote the full trial protocol. HL, PK and APS reviewed and edited draft versions of the protocol. The Statistics and data analysis sections of the trial protocol were written by SB. LW contributed to the Trial treatment section of the protocol, and SU contributed to the Radiology section. PAL, EFM, MP, KGCS and JCL were the initiators of this trial. All authors took part in reading and final approval of the manuscript.

Funding This trial is funded by the Wellcome Trust via an investment in PredictImmune (200448/Z/16/Z). The Apex Healthcare Consulting survey and biomarker development work described herein was funded by a Wellcome Trust Interim Translational Award (099450/Z/12/Z). The trial is cosponsored by Cambridge University Hospitals NHS Foundation Trust and University of Cambridge. The sponsors are not involved in study design; collection, management, analysis, and interpretation of data; writing of the report; and the decision to submit the report for publication. EFM and JCL are supported by Wellcome Trust Intermediate Clinical Fellowships (104064/Z/14/Z and 105920/Z/14/Z respectively).

Competing interests PAL, EFM, KGCS and JCL are coinventors on a patent covering the method of assessing prognosis in IBD. PAL, EFM and KGCS are cofounders and consultants for Predictlmmune. PK and APS are employees of Predictlmmune. JCL is a consultant for Predictlmmune.

Patient consent Not required.

Ethics approval East of England Cambridge South Research Ethics Committee.

Provenance and peer review Not commissioned; peer reviewed for ethical and funding approval prior to submission.

Open access This is an open access article distributed in accordance with the Creative Commons Attribution 4.0 Unported (CC BY 4.0) license, which permits others to copy, redistribute, remix, transform and build upon this work for any purpose, provided the original work is properly cited, a link to the licence is given, and indication of whether changes were made. See: https://creativecommons.org/ licenses/by/4.0/.

\section{REFERENCES}

1. Kaplan GG, Ng SC. Understanding and preventing the global increase of inflammatory bowel disease. Gastroenterology 2017;152:313-21.

2. $\mathrm{Ng} \mathrm{SC}$, Shi HY, Hamidi N, et al. Worldwide incidence and prevalence of inflammatory bowel disease in the 21st century: a systematic review of population-based studies. Lancet 2018;390:2769-78.

3. D'Haens G, Baert F, van Assche G, et al. Early combined immunosuppression or conventional management in patients with newly diagnosed Crohn's disease: an open randomised trial. The Lancet 2008:371:660-7.

4. Schreiber S, Reinisch W, Colombel JF, et al. Subgroup analysis of the placebo-controlled CHARM trial: increased remission rates through 3 years for adalimumab-treated patients with early Crohn's disease. $J$ Crohns Colitis 2013;7:213-21.

5. Hanauer SB, Feagan BG, Lichtenstein GR, et al. Maintenance infliximab for Crohn's disease: the ACCENT I randomised trial. The Lancet 2002;359:1541-9.

6. Colombel JF, Sandborn WJ, Reinisch W, et al. Infliximab, azathioprine, or combination therapy for Crohn's disease. N Engl J Med 2010;362:1383-95.

7. Khanna R, Bressler B, Levesque BG, et al. Early combined immunosuppression for the management of Crohn's disease (REACT): a cluster randomised controlled trial. The Lancet 2015;386:1825-34

8. Sans M, Román AL, Esteve M, et al. Early Use of azathioprine has a steroid sparing effect on recently diagnosed crohn's disease patients. Gastroenterology 2011;109:S-109.

9. Cosnes J, Bourrier A, Laharie D, et al. Early administration of azathioprine vs conventional management of Crohn's Disease: a randomized controlled trial. Gastroenterology 2013;145:758-65.

10. Hart AL, Lomer M, Verjee A, et al. What are the top 10 research questions in the treatment of inflammatory bowel disease? A priority setting partnership with the james lind alliance. Journal of Crohn's and Colitis 2017;11:204-11.

11. Lee JC, Lyons PA, McKinney EF, et al. Gene expression profiling of CD8+ T cells predicts prognosis in patients with Crohn disease and ulcerative colitis. J Clin Invest 2011;121:4170-9.

12. McKinney EF, Lyons PA, Carr EJ, et al. A CD8+ T cell transcription signature predicts prognosis in autoimmune disease. Nat Med 2010;16:586-91.

13. McKinney EF, Lee JC, Jayne DR, et al. T-cell exhaustion, costimulation and clinical outcome in autoimmunity and infection. Nature 2015;523:612-6.

14. Lee J, Biasci D, Noor N, et al. PROFILE trial: predicting outcomes for Crohn's disease using a molecular biomarker. Gut 2017;66:A22-3.

15. Freidlin B, McShane LM, Korn EL. Randomized clinical trials with biomarkers: design issues. J Natl Cancer Inst 2010;102:152-60.

16. Potti A, Mukherjee S, Petersen R, et al. A genomic strategy to refine prognosis in early-stage non-small-cell lung cancer. $N$ Engl $\mathrm{J}$ Med 2006;355:570-80.

17. Gomollón F, Dignass A, Annese V, et al. 3rd European Evidencebased Consensus on the diagnosis and management of crohn's disease 2016: Part 1: diagnosis and medical management. J Crohns Colitis 2017;11:3-25.

18. Su C, Lewis JD, Goldberg B, et al. A meta-analysis of the placebo rates of remission and response in clinical trials of active ulcerative colitis. Gastroenterology 2007;132:516-26.

19. Heap GA, Weedon MN, Bewshea CM, et al. HLA-DQA1-HLA-DRB1 variants confer susceptibility to pancreatitis induced by thiopurine immunosuppressants. Nat Genet 2014;46:1131-4.

20. Bretz F, Maurer W, Brannath W, et al. A graphical approach to sequentially rejective multiple test procedures. Stat Med 2009;28:586-604.

21. Rutgeerts P, Van Assche G, Sandborn WJ, et al. Adalimumab induces and maintains mucosal healing in patients with crohn's disease: data from the EXTEND trial. Gastroenterology 2012;142:1102-11.

22. Baert F, Moortgat L, Van Assche G, et al. Mucosal healing predicts sustained clinical remission in patients with early-stage Crohn's disease. Gastroenterology 2010;138:463-8.

23. Feagan BG, Sandborn WJ, D'Haens G, et al. The role of centralized reading of endoscopy in a randomized controlled trial of mesalamine for ulcerative colitis. Gastroenterology 2013;145:149-57.

24. Feagan B, Sandborn WJ, Rutgeerts P, et al. Performance of crohn's disease clinical trial endpoints based upon different cutoffs for patient reported outcomes or endoscopic activity: Analysis of EXTEND data. Inflamm Bowel Dis 2018;24:1-11.

25. Daperno M, D'Haens G, Van Assche G, et al. Development and validation of a new, simplified endoscopic activity score for Crohn's disease: the SES-CD. Gastrointest Endosc 2004;60:505-12.

26. Khanna R, Zou G, D'Haens G, et al. Reliability among central readers in the evaluation of endoscopic findings from patients with crohn's disease. Gut 2016;65:1119-25.

27. Reinisch W, Colombel JF, D'Haens G, et al. Characterisation of mucosal healing with adalimumab treatment in patients with moderately to severely active crohn's disease: results from the EXTEND trial. J Crohns Colitis 2017;11:425-34.

28. Rimola J, Rodriguez S, García-Bosch O, et al. Magnetic resonance for assessment of disease activity and severity in ileocolonic Crohn's disease. Gut 2009;58:1113-20.

29. Rimola J, Ordás I, Rodriguez S, et al. Magnetic resonance imaging for evaluation of Crohn's disease: validation of parameters of severity and quantitative index of activity. Inflamm Bowel Dis 2011;17:1759-68.

30. Ordás I, Rimola J, Rodríguez S, et al. Accuracy of magnetic resonance enterography in assessing response to therapy and mucosal healing in patients with Crohn's disease. Gastroenterology 2014;146:374-82.

31. Jairath V, Ordas I, Zou G, et al. Reliability of measuring ileocolonic disease activity in crohn's disease by magnetic resonance enterography. Inflamm Bowel Dis 2018;24:440-9.

32. Chan AW, Tetzlaff JM, Altman DG, et al. SPIRIT 2013 statement: defining standard protocol items for clinical trials. Ann Intern Med 2013;158:200-7. 